\title{
Thixotropic Phenomena in Water: Quantitative Indicators of Casimir-Magnetic Transformations from Vacuum Oscillations (Virtual Particles)
}

\author{
Michael A. Persinger \\ Biomolecular Sciences and Behavioural Neuroscience Programs, Laurentian University, Sudbury, \\ ON P3E 2C6, Canada; E-Mail: mpersinger@laurentian.ca
}

Academic Editor: Kevin H. Knuth

Received: 15 July 2015 / Accepted: 28 August 2015 / Published: 7 September 2015

\begin{abstract}
The $\sim 1.5 \times 10^{-20} \mathrm{~J}$ which is considered a universal quantity and is associated with the movement of protons in water also relates to the ratio of the magnetic moment of a proton divided by its unit charge, multiplied by viscosity and applied over the O-H distance. There is quantitative evidence that thixotropy, the "spontaneous" increased viscosity in water when undisturbed, originates from the transformation of virtual particles or vacuum oscillations to real states through conversion of Casimir-magnetic energies that involve the frequency of the neutral hydrogen line and the upper bound threshold value for intergalactic magnetic fields. The results indicate that $1 / 2$ of a single electron orbit is real (particle) and the other $1 / 2$ is virtual (wave). The matter equivalent per $s$ for virtual-to-real states for electrons in $1 \mathrm{~mL}$ of water with a neutral $\mathrm{pH}$ is consistent with the numbers of protons $\left(\mathrm{H}^{+}\right)$and the measured range of molecules in the coherent domains for both width and duration of growth and is similar to widths of intergalactic dust grains from which planets and stars may condense. The de Broglie momentum for the lower boundary of the width of coherent domains multiplied by the fine structure velocity of an electron is concurrent with the quantum when one proton is being removed from another and when the upper boundary of the rest mass of a photon is transformed by the product of velocities for putative "entanglement" and light. Theoretical and experimental results indicate that components of thixotropy, such as specific domains of intercalated water molecules, could display excess correlations over very large distances. Because the energies of the universal quantity and water converge it may be a special conduit for discrete transformations from virtual to real states.
\end{abstract}


Keywords: thixotrophy; entropy; Casimir energy; magnetic energy; intergalactic magnetic fields; water; vacuum oscillations; hydrogen line; electron spin-orbit moment

\section{Introduction}

The thixotropic phenomenon of water may determine many of the parameters that both create and constrain the conditions for living systems. Thixotropy is an emergent property of liquids and gels whose viscosity slowly increases and appears to involve the intrinsic ordering of large numbers of water molecules. Vybiral and Voracek's study [1] of water containing ions indicated that the gel-like behavior developed "spontaneously" over time when undisturbed in closed vessels. Mechanical stimuli dissipated the enhanced viscosity and the correlative properties. The history and primary explanations for the thixotropic phenomenon of water have been reviewed recently by Verdel and Bukovec [2]. They concluded that thixotropy is one "of the more complex characteristics associated with the behaviour of non-Newtonian liquids". One would expect the mechanisms to be relatable to quantum levels of discourse. Here I present quantitative evidence that thixotropic properties of water could reflect a universal interface for the transformation of virtual particles from zero-point, vacuum oscillations to real particles.

The hypothesis by Verdel et al. [3] that thixotropy and proton transfer within water, that is the dynamics of the hydronium ion involving Grotthuss-like mechanisms, are intricately related is revealing. They examined the possibility that a structured network of hydrogen bonds between water molecules and ions in aqueous solutions when left undisturbed for protracted periods near hydrophilic surfaces facilitated this condition. If this were valid, then weak magnetic fields of the appropriate temporal configuration could be contained or "trapped" within these structure networks as predicted by Del Giudice and Preparata [4]. When we [5] exposed spring water containing physiological concentrations of ions to $3 \mathrm{~ms}$ point duration weak magnetic fields in the dark, undisturbed for more than two weeks, spectrophotometry measured a marked increase $(20 \%, \sim 100$ counts) in fluorescence within the 400 to $450 \mathrm{~nm}$ band. If the water was disturbed excessively (during transport to the fluorescent spectrophotometer) or the duration was less than three days this effect was not observed. The $3 \mathrm{~ms}$ point duration had been selected by Koren et al. [6] based upon the solution for the time required for a proton to expand one Planck's length according to a Hubble expansion parameter.

What was unique about this experiment was there were two identically constructed coils between which the water volumes were exposed. Although the peak wavelength was $399 \mathrm{~nm}$ for the activated coil (directly by electric current) compared to the $381 \mathrm{~nm}$ peak for the control samples, the non-activated coil which exhibited the characteristics of a "virtual field" displayed a longer peak wavelength of $409 \mathrm{~nm}$. The difference between the peak fluorescent emissions from water that had been exposed undisturbed in the dark for more than two weeks to the activated and the non-activated (or "virtual" field) coil was $\sim 1.4 \times 10^{-20} \mathrm{~J}$. Subsequent spectral analyses of the successive $1 \mathrm{~nm}$ increments of this band exhibited a clear periodicity at $10 \mathrm{~nm}$, the width of a cell membrane. Neither the enhanced photon emissions nor this spectral profile was displayed by the same volumes of double distilled water exposed to the same conditions. 
The energy associated with the increment at $10 \mathrm{~nm}$ was calculated to be $\sim 1.5 \times 10^{-20} \mathrm{~J}$. This was an important value for several reasons [7]. First, it is consistent with Woutersen and Bakker's [8] measurements of the resonant intermolecular transfer of excitations from distention of $\mathrm{OH}$ excitations over large numbers of water molecules. When the magnetic moment of a proton $\left(1.41 \times 10^{-26} \mathrm{~A} \cdot \mathrm{m}^{2}\right)$ is divided by the unit charge $\left(1.6 \times 10^{-19} \mathrm{~A} \cdot \mathrm{s}\right)$ a diffusivity term emerges with a value of $0.88 \times 10^{-7} \mathrm{~m}^{2} \cdot \mathrm{s}^{-1}$. When applied to the viscosity of water around $25{ }^{\circ} \mathrm{C}\left(8.94 \times 10^{-4} \mathrm{~kg} \cdot \mathrm{m}^{-1} \cdot \mathrm{s}^{-1}\right)$ the force is $7.87 \times 10^{-11} \mathrm{~kg} \cdot \mathrm{m} \cdot \mathrm{s}^{-2}$. If this force is applied across the distance of two $\mathrm{OH}$ bonds $\left(\sim 1.92 \times 10^{-10} \mathrm{~m}\right)$ the available energy would be $\sim 1.5 \times 10^{-20} \mathrm{~J}$ [9]. This quantity of energy is the value between two potassium ions whose bulk interfacial concentrations have been considered the source of the resting potential of the plasma membrane in mammalian cells as well as the net change of $\sim 120 \mathrm{mV}$ (the axonal action potential) upon a unit charge. It is also within measurement error of the second shell hydrogen bonds that have been shown to contribute to the proton's mobility in water [10].

The movement of the proton through water is primarily associated with the presence of the hydronium ion. The average life time of a hydronium ion is about a picosecond, although DeCoursey's brilliant review [10] indicates the experimental range has been between 0.24 to 3 ps. When the average $\left(1 \times 10^{-12} \mathrm{~s}\right)$ is multiplied by the ratio of the proton's magnetic moment to its unit charge, the resulting value is $8.8 \times 10^{-20} \mathrm{~m}^{2}$, or, a functional length of $0.297 \mathrm{~nm}$. The actual distance between water molecules is measured to be around $0.29 \mathrm{~nm}$. This identical congruence strongly suggests that the duration of the hydronium ion, defined as the transient sequestering of the proton, is actually determined by the diffusivity of the dynamics of water. Alternatively the duration of the hydronium ion phenomena is a function of the specific distance (space) between source molecules.

If the mobile proton is a contributory source to thixotropy and the proton and an electron, the neutral hydrogen atom, constitute $90 \%$ of the matter of the universe as inferred by the hydrogen line of $1.42 \mathrm{GHz}$, then the properties of thixotropy should share commonalities with this fundamental matter. The spatial dimensions of the ordered domains of water vary depending upon the experimental procedure. Bunkin's [11] spherical gas cavities, created by dissolving atmospheric gas into ion-containing water, displayed radii between $10 \mathrm{~nm}$ and $100 \mathrm{~nm}$. Clusters of these units expand to a radius of $0.5 \mu \mathrm{m}$. Incident radiant energy converted the water vesicles into vapour bubbles [12]. For a variety of chemical systems that included acetic acid, D-glucose, $\mathrm{NaCl}$ and ethanol within double-distilled water Sedlak [13] found that the number of solute molecules per domain ranged from $10^{3}$ to $10^{8}$. More importantly from the perspective of etiology the developments of the supramolecular structures were a function of time and exhibited a temporal asymptote. After about 80 days the domain structures appeared stable with most sizes between $\sim 30 \mathrm{~nm}$ and $0.5 \mu \mathrm{m}$ with an average of about $100 \mathrm{~nm}$.

As reported by Pagani et al. [14] spaces defined as dust grains with an average size of $0.1 \mu \mathrm{m}$ $(100 \mathrm{~nm})$ are the primary constituents of cold molecular clouds in the interstellar medium. These clouds and their materials contribute (through mechanisms yet to be fully described) to the formation of stars and planets. Estimation of the simple force of the universal set, the known universe, is the product of its mass $\left(\sim 10^{52} \mathrm{~kg}\right)$, its length $\left(\sim 10^{26} \mathrm{~m}\right)$ and the square of the zero point vacuum oscillation, i.e., $10^{86} \mathrm{~s}^{-2}$. That value is $\sim 10^{164} \mathrm{~N}$ [15]. Assuming the smallest volume unit to be a Planck's voxel $\left(10^{-105} \mathrm{~m}^{2}\right)$ and the volume of the universe to be $10^{78} \mathrm{~m}^{3}$ here would be $10^{183}$ Planck's voxels resulting in $\sim 10^{-19} \mathrm{~N}$ per voxel. Applied across the most frequent space or wavelength, the neutral hydrogen line of $21 \mathrm{~cm}$, the "pervasive" energy would be $10^{-20} \mathrm{~J}$. If current coefficients for the source values are 
included the likely quantity is $\sim 2 \times 10^{-20} \mathrm{~J}$. The similarity of punctate spaces of formative matter and this energy to the structured organization and energies contributing to the proton within water suggests a commonality that could originate from fundamental forces where virtual and real particles interact and transform from and into entropy.

\section{Quantum Casimir-Macroscopic Magnetic Field Energies}

The Casimir effect has been considered a "pure quantum effect" from zero-point oscillations. According to Bordag et al. [16] it represents the zero-point energy of a quantized field. To transcend and integrate spatial levels from the smallest space (Planck's Length) the Casimir process should be transformable to macroscopic phenomena, such as matter-level magnetic fields. If this occurs then there should be a convergence of quantitative solutions that relate to the properties of matter within specific boundaries.

Casimir energy is:

$$
\mathrm{E}_{\mathrm{C}}=\pi^{2} 240^{-1} \hbar \mathrm{c} \mathrm{a} \mathrm{a}^{-4}
$$

where $\hbar$ is the modified Planck's constant $\left(1.05 \times 10^{-34} \mathrm{~J} \cdot \mathrm{s}\right), \mathrm{c}$ is the velocity of light in a vacuum, "a" refers to the width between two Casimir plate-like conditions and $\mathrm{m}^{3}$ is functional volume of the separation between the two plates, and, "magnetic field energy" is:

$$
\mathrm{E}_{\mathrm{M}}=\mathrm{B}^{2}(2 \mu)^{-1} \mathrm{~m}^{3}
$$

where $B$ is the strength of the magnetic field, $\mu$ is magnetic permeability (in a vacuum, $4 \pi \times 10^{-7} \mathrm{~N} \cdot \mathrm{A}^{-2}$ ) and $\mathrm{m}^{3}$ is the volume within which the field is contained. When these energies are set equal and the "inter-plate" distance for the idealized Casimir separation is calculated the relationship is:

$$
\left.\mathrm{a}^{4}=\left[\pi^{2} 240^{-1}\right) \hbar c(2 \mu)\right] \cdot \mathrm{B}^{-2}
$$

The volume component is cancelled and the only variable (non-constant) is B, the strength of the field. The candidate to relate the two sources of energy must be pervasive. One possibility is the "universal" wave structure and frequency that is ubiquitous: the neutral hydrogen line. The strength of the B field required to converge with the energy from the neutral hydrogen line $(1.42 \mathrm{GHz})$ or $9.41 \times 10^{-25} \mathrm{~J}$ (the product of frequency and Planck's regular constant, $6.626 \times 10^{-34} \mathrm{~J} \cdot \mathrm{s}$ ) for the magnetic moments of an electron's orbit and spin are quantifiable. These values are $\mu_{\mathrm{B}}=9.2740780 \times 10^{-24} \mathrm{~J} \cdot \mathrm{T}^{-1}$ and $\mu_{\mathrm{e}}=9.2847701 \times 10^{-24} \mathrm{~J} \cdot \mathrm{T}^{-1}$ [17]. For convenience only the first three decimal points will be employed for further analyses.

The magnetic field strength associated with the convergence with the spin or orbit magnetic moment of an electron is $\sim 1.01 \times 10^{-1} \mathrm{~T}$. The resultant value for $\sqrt[4]{a}$ is between 23.7 and $23.8 \mathrm{~nm}$. The Casimir distance (equation 3 ) derived from the equivalence of Casimir and magnetic energy for the electron orbital magnetic moment $\mu_{\mathrm{B}}$ would be $2.373 \times 10^{-8} \mathrm{~m}$. If it were a wavelength of electromagnetic radiations, such as photons, moving at $\mathrm{c}\left(2.998 \times 10^{8} \mathrm{~m} \cdot \mathrm{s}^{-1}\right)$, the time required for one orbit would be $0.792 \times 10^{-16} \mathrm{~s}$. This duration is almost exactly $1 / 2$ of the time for one orbit of an electron around a Bohr atom. With a standard Bohr radius $\left(52.9 \times 10^{-11} \mathrm{~m}\right)$ for an electron moving at the fine structure velocity $\left(2.190 \times 10^{6} \mathrm{~m} \cdot \mathrm{s}^{-1}\right)$ the time is $1.517 \times 10^{-16} \mathrm{~s}$. 
The occurrence of the $1 / 2$ value has significant implications for the transformation of virtual particles to real particles. If the boundary conditions, that is the single orbital completion, depends upon time and is associated with external magnetic fields, than creation of particles could occur [16]. From a strictly geometric perspective, because the movement is within a circle, the packet of energy is perpetually "accelerating". If for a given single orbit of an electron around a proton half of the time the electron occurs as a particle (matter) while the other half of the orbit it operates as an emergent virtual particle (wave) derived from the Casimir-magnetic energy transformation, manifestations of random vacuum fluctuations could occur.

\section{Virtual to Real Particles as Casimir-Magnetic Transformations}

If the single orbit is related by $1 / 2$ to the transformation from virtual manifestations to "real" particles or matter and the changing boundaries of magnetic fields are present there should be creation of particles from the zero point vacuum oscillations. The transformation vector would be the strength of the magnetic field coupled to the geometry of sub-matter space [18]. Neronov and Vovk [19] have calculated that the lower limit for intergalactic magnetic field strengths is in the order of $3 \times 10^{-20} \mathrm{~T}$. According to Kronberg [20] the upper limit for a uniform magnetic field component in the intergalactic medium is $\sim 6 \times 10^{-15} \mathrm{~T}$.

The effect upon the orbital-spin magnetic moments of an electron would be between $2.8 \times 10^{-44} \mathrm{~J}$ for the lower limit and $5.6 \times 10^{-39} \mathrm{~J}$ for the upper limit per orbit. Because the time for each Bohr orbit is $1.52 \times 10^{-16} \mathrm{~s}$ the energy would be $1.83 \cdot 10^{-28} \mathrm{~J} \cdot \mathrm{s}^{-1}$ and $3.68 \times 10^{-23} \mathrm{~J} \cdot \mathrm{s}^{-1}$, respectively. The latter value is significant. The equivalent $\mathrm{kT}$ solution where $\mathrm{k}$ is the Boltzmann constant $\left(1.38 \times 10^{-23} \mathrm{~J} \cdot \mathrm{T}^{-1}\right)$ for this energy is equivalent to $\mathrm{T}=2.7 \mathrm{~K}$ which is identical within measurement error to the Cosmic Microwave Background temperature of $2.725 \mathrm{~K}$. Interestingly, the energy within $1 \mathrm{~m}^{3}$ of space from the upper limit intergalactic medium according to classic formula would be $1.8 \times 10^{-23} \mathrm{~J}$.

The mass equivalent of this quantity of energy associated with the upper limit for the uniform magnetic field within the intergalactic medium is $0.41 \times 10^{-39} \mathrm{~kg}$. This value is within the central range of the upper rest mass of photons at the velocity of light within the different ranges of the electromagnetic spectrum as described by $\mathrm{Tu}$ et al. [21]. For example the values in their Table 1, which summarizes 10 different publications over a period of 60 years, indicated the value is within the upper mass range for photons from short pulse radiation to gamma ray bursts.

The concept of transformations of Casimir to magnetic energies should exhibit equivalents across the levels of scientific discourse within which specific mechanisms should be discernable. For example the differential presence and absence of the magnetic moment $(1,0)$ in the ortho- and para-isomeric forms of water [22] could be contributory. Yada et al. [23] employed THz Time Domain (Attenuated Total Reflection) Spectroscopy to measure the slow/fast relaxation and intermolecular stretching vibration/libration features of the dielectric constants of water. They found that the fast component existed within the sub-picosecond range and exhibited minimal temperature dependence over an approximately $90 \mathrm{~K}$ range that included typical terrestrial surface values.

The fast component had been attributed to the disruption and forming of hydrogen bonds or the inertial motion of water molecules. The fast decay activation energy of hydrogen bonding was $\sim 11.1 \pm 0.4 \mathrm{~kJ}$ per molecule $\left(\sim 1.8 \times 10^{-20} \mathrm{~J}\right.$ per molecule). Yada et al. [23] concluded that the fast 
component was an individual process that transiently appeared within the dynamic hydrogen bonding network and was governed by a collision process. They discerned a fraction $(\sim 0.02)$ of isolated or "free" molecules that occupied the interface between random and non-random distributions. In the balance of probabilities these free molecules would exist as clustered distributions which could maximize the total entropy. These physical conditions and associated quantitative values could be sufficient to encourage Casimir-magnetic field transformations within the types of clusters inferred from other approaches $[3,4,12,15,24]$. It may be relevant that the product of the lower limit for intergalactic magnetic field strength, $3 \times 10^{-20} \mathrm{~T}$ [19], unit charge $\left(1.6 \times 10^{-19} \mathrm{~A} \cdot \mathrm{s}\right)$ and the diffusivity value for light in a vacuum $\left(\sim 9 \times 10^{16} \mathrm{~m}^{2} \cdot \mathrm{s}^{-1}\right)$ is an increment of energy whose equivalent duration (when divided into Planck's constant) is $\sim 1.5 \times 10^{-12} \mathrm{~s}$.

\section{Contribution to Thixotropy}

If the dynamic proton from the hydronium ion is contributing to the transformation of particles, specifically the electron, from vacuum oscillations then there should be a quantitative relationship between the numbers of protons in a volume of water and the electrons required for the emergence from the virtual to real states in order to ensure parity. In $1 \mathrm{cc}$ of water which is $0.056 \mathrm{M}$, the numbers of molecules according to Avogadro's values would be $6.023 \times 10^{23}$ molecules per Mole multiplied by $0.056 \mathrm{M}$ or $3.35 \times 10^{20}$. Assuming a neutral $\mathrm{pH}$ of 7.0 for the water, the numbers of protons would $10^{-7}$ $\mathrm{M}$. Hence the numbers of protons would be $3.35 \times 10^{13} \mathrm{H}^{+}$. If there is a one-to-one transformation between the proton and emerging electron there would be $0.41 \times 10^{-39} \mathrm{~kg}$ per orbit multiplied by $3.35 \times 10^{13}$ reference $\mathrm{H}^{+}$or the equivalence of $1.37 \times 10^{-26} \mathrm{~kg}$. Assuming the rest mass of an electron is $9.11 \times 10^{-31} \mathrm{~kg}$, this would be $0.15 \times 10^{5}$ electrons per second.

From the current perspective this means that per second approximately $1.5 \times 10^{4}$ virtual electrons could be shifted from virtual to real states. The typical numbers of molecules per Coherent Domain [24] of structured water ranges from $10^{3}$ to $10^{8}$. By the time stability of numbers and expansions of domains occurred around 180 days or $1.5 \times 10^{7} \mathrm{~s}$ there would be between $10^{3}$ largest to $10^{8}$ smallest domains per cc. This assumes there have been no energies from mechanical agitation or in some instances ambient photons or lighting to disrupt the process.

If one assumes each transformation from virtual to real states involved the universal quantity of $2 \times 10^{-20} \mathrm{~J}$, there are $0.15 \times 10^{5}$ virtual electron transformations to real particle equivalents per $\mathrm{s}$ and the asymptote is $1.5 \times 10^{7} \mathrm{~s}$ for the process, then the energy available to $1 \mathrm{cc}$ of water would be $4.5 \times 10^{-9} \mathrm{~J}$. Simplistically the relationship in units between viscosity and energy (E) is:

$$
\left(\mathrm{kg} \cdot \mathrm{m}^{-1} \cdot \mathrm{s}^{-1}\right)=\mathrm{E} \cdot\left(\mathrm{m}^{3} \cdot \mathrm{s}^{-1}\right)^{-1}
$$

For $10^{-6} \mathrm{~m}^{3}(1 \mathrm{cc})$ for $1 \mathrm{~s}$ unity $(1 \mathrm{~Hz})$ the viscosity would be $4.5 \times 10^{-3} \mathrm{~Pa} \cdot \mathrm{s}$ which at $20^{\circ} \mathrm{C}$ would be about 5 times more than the typical values of $8.9 \times 10^{-4} \mathrm{~Pa} \cdot \mathrm{s}$. This is within the range of increased viscosity noted in thixotropic reactions $[1,2]$.

If the energy associated with the structuring of water within these domains and any solutes within them occurs as increments of the universal energy value of $\sim 10^{-20} \mathrm{~J}$, then the consequences of these depositions should be also evident. Estimations from Grandbois et al.'s [25] measurement of covalent bond forces for silicon-gold and silicon-carbon was equivalent to about $1.4 \times 10^{-20} \mathrm{~J}$ in their 
preparations which is within the range of the second shell hydrogen energies associated with the movement of protons according to DeCoursey [10]. The shift of wavelength in the Murugan et al. studies [5] in which spring water was undisturbed in the dark for more than two weeks was most for samples within the "virtual fields" and was equivalent to $10^{-20} \mathrm{~J}$. Spectral analyses of this shift in peak fluorescent spectrometry indicated a periodicity of about $10 \mathrm{~nm}$. This is the width of the classical plasma cell membrane as well as the lower limit for the coherent domains noted in water [24].

\section{Determination of Spatial Limits and Boundaries}

One important question is why is the upper limit around $100 \mathrm{~nm}$ for both the coherent domains within thixotropic water and the average particle density in intergalactic space. The most obvious answer is that it reflects some fundamental boundary condition. The energy from the upper limit of the intergalactic medium of $6 \times 10^{-15} \mathrm{~T}$ within a sphere with a diameter of $100 \mathrm{~nm}$ would be $9.4 \times 10^{-45} \mathrm{~J}$. However if this is coupled to the pervasive neutral hydrogen line of $1.42 \times 10^{9} \mathrm{~Hz}$, the energy per $\mathrm{s}$ would be $1.35 \times 10^{-35} \mathrm{~J}$. The mass equivalent of that quantity of energy is $1.5 \times 10^{-52} \mathrm{~kg}$ or exactly within the upper boundary for the rest mass of a photon [21]. Presumably this upper limit for the rest mass of a photon is at the limen of imaginary $(i)$ to real transitions from virtual (vacuum oscillations) entities with properties of particles and waves.

This approach may accommodate the upper limit of coherent domains in water in the dark. In this instance the photon source is from the virtual space of sub-matter. It might also explain the expansion of the larger aggregates of these domains in some experimental preparations. For example Pollack and his colleagues [26-28] have noted that the exclusion zone (EZ) expands during exposure to microscopic lights. When infrared light was removed after only $5 \mathrm{~min}$ of exposure the EZ width remained constant but exhibited fluctuations for about $30 \mathrm{~min}$. During the subsequent $15 \mathrm{~min}$ its width diminished by half. Radiant energy increased widths of the EZs reversibly and in a wavelength-dependent manner. The authors concluded that "incident radiant energy may be stored in water as entropy loss and charge separation". Pollack's EZs [28] could be considered a higher order aggregate of thixotropy which incorporates the geometric properties of two adjacent planes or "plates". One of the plates would be the interfacial water, the other plate would be the surface of the material and the "plane" or "shell" of protons.

The lower limit of about $20 \mathrm{~nm}$ for the coherent domains should also reflect cosmological boundaries. I [29] have considered the "density" of photon-equivalents if all matter were considered in this context. Assuming the current mass of the universe to be about $2 \times 10^{52} \mathrm{~kg}$ [30] which is similar to values derived from both empirical and theoretical estimates and the upper limit of the rest mass of the photon to be $\sim 2 \times 10^{-52} \mathrm{~kg}$, the numbers of photon equivalents are $10^{104}$ photons per universe. Assuming the median value for the three-dimensional spatial volume of the universe to be $10^{78} \mathrm{~m}^{3}$ there would be $10^{-26} \mathrm{~m}^{3}$ per photon. If the volume were symmetrical the width would be 10 to $20 \mathrm{~nm}$. This is the lower limit of the coherent domains. Obviously there are alternative explanations for the minimum and maximum values for the coherent domains. There have been many suggestions by thoughtful experimenters and theorists [31-33]. One would expect their explanations to compliment and extend the current calculations rather than refute them. 


\section{The Issue of Entanglement}

The principle of superposition indicates that a new state of a system may be composed of two or more states such that the new state shares components of the properties of each of the combined states [34]. It is consistent with de Broglie's concept of "matter waves": every particle in the universe is associated with a wave propagating through space. When a quantum system contains more than one particle the superposition potential results in "entanglement". It can also be expressed empirically as an excess correlation between the behaviour of particle/waves within two loci separated by any distance. When Mach's Principle [35] is applied the expansiveness of excess correlation becomes apparent. It states that "the law of causality is sufficiently characterized by saying that it is the presupposition of mutual dependence of phenomena" and "every phenomenon is a function of other phenomena and of certain spatial and temporal positions".

We have demonstrated experimentally that photon emissions from chemiluminescent reactions [36], shifts in $\mathrm{pH}$ [37] within spring water and the viability of malignant cells in culture [38] located in spaces separated by $10 \mathrm{~m}$ to $3 \mathrm{~km}$ displayed excess correlations under conditions that were consistent with a non-zero rest mass for photons [21]. However the excess correlations were only manifested when the reactions within the two volumes of water occurred in the centers of rotating, phase-modulated magnetic fields that shared the same changing angular velocities. When a coupled decreasing phase/frequency-modulated pattern and accelerating group velocity pattern was followed by a coupled increasing phase/frequency modulated pattern immersed in decreasing group velocity the powerful excess correlation occurred. The duration of the excess correlation was about 7 to $8 \mathrm{~min}$. Reverse order presentations or fixed angular velocities did not produce the effect.

Ernst Mach presumed the connection to produce the condition such that the behavior of any part of the cosmos was determined by all of its parts was associated with shared momentums, $p$. If de Broglie's pilot waves are applicable at quantum levels then $p=\mathrm{h} \cdot \lambda^{-1}$ where the former is Planck's constant and the latter is wavelength. If this were generalized to the $10 \mathrm{~nm}$ to $100 \mathrm{~nm}$ width of cohesive zones as wavelengths the momentum would range from $6.626 \times 10^{-26}$ to $6.626 \times 10^{-27} \mathrm{~kg} \cdot \mathrm{m} \cdot \mathrm{s}^{-1}$ and when enhanced to the fine structure velocity $\left(2.19 \times 10^{6} \mathrm{~m} \cdot \mathrm{s}^{-1}\right)$ of the electron the energy would be between $1.5 \times 10^{-19} \mathrm{~J}$ to $1.5 \times 10^{-20} \mathrm{~J}$. This extends over a band of wavelengths between $\sim 1.36 \mu \mathrm{m}$ to $\sim 13.6 \mu \mathrm{m}$. The lower end of the band includes the wavelengths $(2.9-3.3 \mu \mathrm{m})$ where liquid water absorbs most strongly and corresponds to the O-H stretching mode [26,27]. It may not be spurious that Bohr's original frequency [39] for the quantum involved with one proton being removed from another, based upon $1.32 \cdot \omega_{0} \sqrt{ }\left(\mathrm{m} \cdot \mathrm{M}^{-1}\right)$ where $\mathrm{m}$ and $\mathrm{M}$ are the mass of the electron and proton respectively and $\omega_{0}$ is $6.2 \times 10^{15} \mathrm{~Hz}$, is $\lambda=1.57 \mu \mathrm{m}$.

However for excess correlations to occur "simultaneously" from our temporal perspective within the universal boundaries there must be an additional velocity whose value ultimately connects the upper boundaries for photon masses to the energies $\left(\sim 10^{-20} \mathrm{~J}\right)$ congruent with the operations within water that would contribute to increased viscosity. Persinger and Koren [40] equated the product of the four dimensional geometries for circularity, that is, $21.3 \pi^{4} \mathrm{r}^{7} \mathrm{f}$ with the aggregate $\mathrm{m}^{7} \cdot \mathrm{s}^{-1}$, to the optimal combination of universal values that balanced this relationship. They were $\mathrm{G}^{2} \cdot \mathrm{m}^{2} \cdot \mathrm{d} \cdot \mathrm{t}^{3}$, where $\mathrm{G}$ was the Newtonian Gravitational constant, $\mathrm{m}$ was the mass of the universe, $\mathrm{d}$ was its diameter and $\mathrm{t}$ was its age. The resulting value for this "diffusivity" term for the 7 th $\operatorname{root}$ was $2.84 \times 10^{23} \mathrm{~m} \cdot \mathrm{s}^{-1}$. They called 
this value the "entanglement" velocity. Approximately 7 to 8 min would be required for the universe to be traversed.

A process with this velocity moving between the earth and the sun would require $\sim 10^{-12} \mathrm{~s}$ or the life-time of a hydronium ion before the proton moves to the next water molecule. The temperature equivalent for $2 \times 10^{-20} \mathrm{~J}$ according to the $\mathrm{kT}$ relationship to energy where $\mathrm{k}$ is the Boltzmann constant $\left(1.38 \times 10^{-23} \mathrm{~J} \cdot \mathrm{K}^{-1}\right)$ would be $1400 \mathrm{~K}$. The recent measurement of copious water molecules on the solar surface [41] but only within the umbra of sunspots where $\mathrm{T}<4000 \mathrm{~K}$ suggests that "excess" correlation of specific dynamics could occur between solar states and those within terrestrial water. If this were valid, then the explanations for the historical variations [31] in relative viscosities of aqueous solutions that vary with the solar cycle, such as the Piccardi effect [42], may have alternative explanations.

Additional analyses [43] indicated that the ratio of the total energy within the universe expressed as a magnetic field, derived from equation (2) and the total electric field in $\mathrm{V} \cdot \mathrm{m}^{-1}$ resulted in a similar order of magnitude of $\sim 0.9 \times 10^{23} \mathrm{~m} \cdot \mathrm{s}^{-1}$. When the two equations that had produced similar velocities were set equal, the value for the frequency required for an identity to occur between the two was $\sim 10^{-1} \mathrm{~Hz}$. This is an interesting derivation because the classical energy of the upper boundary of the rest mass of a photon $\left(2 \times 10^{-52} \mathrm{~kg}\right)$ from multiplying by the square of the velocity of light is $1.8 \times 10^{-35} \mathrm{~J}$. The "frequency" of this quantity when divided by Planck's constant is within this range. The conclusion [42] was that "diffusion velocity" derived from the appropriate combination of $G$, and various powers of the width of the universe, its mass, and its age is related to the velocity produced by the ratio of the voltage field to the magnetic field by a frequency that at the quantum level is equivalent to the upper rest mass of the photon.

The entanglement of fundamental entities with particle-wave properties, such as the photon, could contribute to the quantum of universal energy $\left(10^{-20} \mathrm{~J}\right)$ that may be central to the coherent domains which emerge in thixotropic phenomena. If the upper limit of the rest mass photon is $\sim 2 \times 10^{-52} \mathrm{~kg}$ [21], and it is considered imaginary $(i)$ and the diffusivity velocity of $2.8 \times 10^{23} \mathrm{~m} \cdot \mathrm{s}^{-1}$ is considered imaginary then the product of the two becomes a real number. If multiplied by the velocity of light $\left(3 \times 10^{8} \mathrm{~m} \cdot \mathrm{s}^{-1}\right)$ the resulting energy is $1.8 \times 10^{-20} \mathrm{~J}$. This quantity [10] is consistent with the second shell hydrogen bonds and experimental values for mobility of protons in water.

Experimental verification discerns if the concept is operational within specific application geometries. The conditions for "excess correlation" or "entanglement" may exist within one of the most ubiquitous sized life forms: bacteria. According to Pischalnikov, Pershin and Bunkin [44] the transient coherent modulations of absorption spectra isolated from purple bacteria were due to rotational resonances of the ortho- $\mathrm{H}_{2} \mathrm{O}$ isomer that exhibits the magnetic moment. They concluded that interaction of rotational modes with the transfer states for charge produced coherent kinetic modulations. If these rotational modes exhibited magnetic fields with changing angular velocities that were tuned variants of those which produced the conspicuous excess correlation for photon radiant flux densities in our experimental settings [36] then the central role of one of the specific heterogeneities of water could also be tested empirically. The validity of this condition could significantly alter the explanations for interactions between physical chemical reactions in water particularly in thixotropic environments.

Similar quantities of energies within the water molecule and across levels of discourse from the photon to intergalactic magnetic fields when coupled partially by the entanglement velocity indicate 
the prominence of the value of $10^{-20} \mathrm{~J}$ as the energy metric for both dynamics and structure within the space that is occupied by all matter including water. It might be considered the conduit by which energy and virtual particles transform from and to entropy. Pagani et al. [14] measured the interstellar dust particles and found that the clusters in the core of the aggregates absorbed background radiation at $8 \mu \mathrm{m}$. Assuming the velocity of light the frequency is $0.38 \times 10^{14} \mathrm{~Hz}$ and the equivalent energy is $2.4 \times 10^{-20} \mathrm{~J}$. The extinction curve for the scattered light from these cores of 0.1 to $1 \mu \mathrm{m}$ particles within the interstellar medium indicated the source was from the dense parts of the molecular cloud cores.

Pagani et al. [14] hypothesized that the scattered light within the near infrared could arise from stochastically heated small dust grains such as polycyclic hydrocarbons but there would be an additional requirement for exposure to UV and the optical interstellar radiation field whose current values were not considered sufficient. There may be an alternative possibility. If this involved the transformation of virtual to real particles, based upon the argument developed in Sections 3 and 4 and was typical of the dichotomy attributable to kinetic and potential energies, $1 / 2$ of that energy would be distributed more or less equally between the virtual (wave) and particle state. Within water where the bond energies are prominently $\sim 1.5 \times 10^{-20} \mathrm{~J}$ and the value for proton movement are comparable the conditions would be more favorable to direct transformation from virtual to real matter.

\section{Conclusions}

The persistent, subtle, and specific properties of thixotropic phenomena in water meet the quantitative criteria for a special condition that could be distributed throughout the sub-matter spatial fabric of the universe. The prevalence of the quantity of energy in the order of $2 \times 10^{-20} \mathrm{~J}$ derived from the product of the ratio of the proton's magnetic moment to its unit charge and viscosity applied across the O-H length could mediate the dynamics that connect water to the transformation of vacuum oscillations (or virtual particles) to real states and determine the spatial and temporal boundaries of the coherent domains that create the thixotropic state.

The state could originate from the interaction between the spin-orbit magnetic moments and neutral hydrogen line frequency that sets the condition for a single orbit to display $1 / 2$ wave (virtual) and $1 / 2$ real (particle) properties. The mass equivalent of the transformation from virtual to real states from energy converging between Casimir-magnetic field strengths at the limen of the intergalactic background magnetic field compliments the numbers of free protons $(\mathrm{pH})$ in an optimal volume of water and determines the numbers of interactive molecules within the domains. When undisturbed or within minimal radiant influence the conduit of $\sim 2 \times 10^{-20} \mathrm{~J}$ would conservatively increase the viscosity to five times the usual value.

The involvements of the energies that reflect shifts of photons from rest mass as well as a "diffusivity" or "entanglement" velocity that converge with $10^{-20} \mathrm{~J}$ as a universal value emerge across levels of spatial structure and suggest that thixotropic conditions of water could reflect universal conditions. The occurrence of excess correlations between solar and terrestrial water molecules could accommodate the periodicities noted in thixotropic responses reported in various chemical systems over the previous decades. 


\section{Acknowledgments}

This document is dedicated to E. Del Giudice, Gerald H. Pollack and the many original thinkers who have confronted the "slings and arrows of outrageous fortune" to pursue the truth.

\section{Conflict of Interests}

The author declares no conflict of interest.

\section{References}

1. Vybiral, B.; Voracek, P. Long Term Structural Effects in Water: Autothixotropy of Water and Its Hysteresis. Homeopathy 2007, 96, 183-188.

2. Verdel, N.; Bukovec, P. Possible Further Evidence for the Thixotropic Phenomenon of Water. Entropy 2014, 16, 2146-2160.

3. Verdel, N.; Jerman, I.; Bukovec P. The "Autothixotropic" Phenomena of Water and Its Role in Proton Transfer. Int. J. Mol. Sci. 2011, 12, 7481-7491.

4. Del Giudice, E.; Preparata, G. Coherent Dynamics in Water as a Possible Explanation of Biological Membranes Formation. J. Biol. Phys. 1994, 20, 105-116.

5. Murugan, N.J.; Karbowski, L.M.; Lafrenie, R.M.; Persinger, M.A. Maintained Exposure to Spring Water but not Double Distilled Water in Darkness and Thixotropic Conditions to Weak $(\sim 1 \mu \mathrm{T})$ Temporally Patterned Magnetic Fields Shift Photon Spectroscopic Wavelengths: Effects of Different Shielding Materials. J. Biophys. Chem. 2015, 16, 14-28.

6. Koren, S.A.; Dotta, B.T.; Persinger, M.A. Experimental Photon Doubling as a Possible Local Inference of the Hubble Parameter. Open Astron. J. 2014, 7, 1-6.

7. Persinger, M.A. 10-20 Joules as a Neuromolecular Quantum in Medicinal Chemistry: An Alternative Approach to Myriad Molecular Pathways. Cur. Med. Chem. 2010, 17, 3094-3098.

8. Woutersen, S.; Bakker, H.J. Resonant Intermolecular Transfer of Vibrational Energy in Liquid Water. Nature 1999, 402, 507-509.

9. Karbowski, L.M.; Persinger, M.A. Variable Viscosity of Water as the Controlling Factor in Energetic Quantities that Control Living Systems: Physicochemical and Astronomical Interactions. Int. Lett. Chem. Phys. Astron. 2014, 4, 1-9.

10. Decoursey, T.E. Voltage-gated Proton Channels and Other Proton Transfer Pathways. Physiol. Rev. 2003, 83, 475-579.

11. Bunkin, N.F.; Bunkin, F.V. Screening of Strongly Charged Macroparticles in Liquid Electrolyte Solutions. J. Exp. Theor. Phys. 2003, 96, 730-746.

12. Pollack, G.H. The Fourth Phase of Water: Beyond Solid, Liquid and Vapor; Ebner and Sons: Seattle, WA, USA, 2013.

13. Sedlak, M. Real-Time Monitoring of the Origination of Multimacroion Domains in Polyelectrolyte Solution. J. Chem. Phys. 2005, 122, 151102.

14. Pagani, L.; Stinacker, J.; Bacmann, A.; Stutz, A.; Henning, T. The Ubiquity of Micrometer-Sized Dust Grains in the Dense Interstellar Medium. Science 2010, 329, 1622-1624. 
15. Persinger, M.A. Quantitative Convergence between Physical-Chemical Constants of the Proton and the Properties of Water: Implications for Sequestered Magnetic Fields and a Universal Quantity. Int. Lett. Chem. Phys. Astron. 2014, 2, 1-10.

16. Bordag, M.; Mohideen, U.; Mostepanenko, V.M. New Developments in the Casimir Effect. Phys. Rep. 2001, 353, 1-205.

17. Illingworth, V. (Ed.) The Penguin Dictionary of Physics; Penguin Books: London, UK, 1990.

18. Persinger, M.A. Discrepancies between Predicted and Observed Intergalactic Magnetic Field Strengths from the Universe's Total Energy: Is It Contained within the Submatter Spatial Geometry? Int. Lett. Chem. Phys. Astron. 2014, 11, 18-23.

19. Neronov, A.; Vovk, I. Evidence of Strong Extragalactic Magnetic Fields from Fermi Observations of TeV Blazars. Science 2010, 328, 73-78.

20. Kronberg, P.P. Extragalactic Magnetic Fields. Rep. Prog. Phys. 1994, 57, 325-382.

21. Tu, L.-C.; Luo, J.; Gilles, G.T. The Mass of the Photon. Rep. Prog. Phys. 2005, 68, 77-130.

22. Tikhonov, V.I.; Volkov, A.A. Separation of Water into Its Ortho and Para Isomers. Science 2002, 296, 2363.

23. Yada, H.; Nagai, M.; Tanaka, K. Origin of the Fast Relaxation Component of Water and Heavy Water Revealed by Terahertz time-Domain Attenuated Total Reflection Spectroscopy. Chem. Phys. Lett. 2008, 464, 166-170.

24. Del Giudice, E.; Spinetti, P.R.; Tedeschi, A. Water Dynamics at the Root of Metamorphosis in Living Organisms. Water 2010, 2, 566-586.

25. Grandbois, M.; Beyer, M.; Rief, M.; Clausen-Schaumann, H.; Gaub, H.E. How Strong Is a Covalent Bond? Science 1999, 283, 1727-1730.

26. Zheng, J-M.; Chin, W-C.; Khijniak, E.; Khijniak, E., Jr.; Pollack, G.H. Surfaces and Interfacial Water: Evidence that Hydrophilic Surfaces Have Long-Range Impact. Adv. Colloid Interface Sci. 2006, 127, 19-27.

27. Chai, B.; Yoo, H.; Pollack, G.H. Effect of Radiant Energy on Near-Surface Water. J. Phys. Chem. B 2009, 113, 13953-13958.

28. Pollack, G.H.; Figueroa, X.; Zhao, Q.; Molecules, Water, and Radiant Energy: Clues for the Origin of Life. Int. J. Mol. Sci. 2009, 10, 1419-1429.

29. Persinger, M.A. Convergence of Numbers of Synapses and Quantum Foci within the Human Brain Space: Quantitative Implications of the Photon as the Source of Cognition. Int. Lett. Chem. Phys. Astron. 2014, 11, 59-66.

30. Persinger, M.A. A Simple Estimate for the Mass of the Universe: Dimensionless Parameter A and the Construct of "Pressure". J. Phys. Astrophys. Phys. Cosmol. 2009, 3, 1-3.

31. DeMeo, J. Water as a Resonant Medium for Unusual External Environmental Factors. Water 2011, 3, 1-47.

32. Voeikov, V.L.; Del Giudice, E. Water Respiration-The Basis of the Living State. Water 2009, 1, 52-75.

33. Davidson, R.M.; Lauritzen, A.; Seneff, S. Biological Water Dynamics and Entropy: A Biophysical Origin of Cancer and Other Diseases. Entropy 2013, 15, 3822-3876.

34. Aczel, A.D. Entanglement: The Greatest Mystery in Physics; Basic Books: New York, NY, USA, 2002. 
35. Mach, E.; McCormack, T.J. The Science of Mechanics: A Critical and Historical Account of Its Development; Open Court Publishing Company: Chicago, IL, USA, 1988.

36. Dotta, B.T.; Persinger, M.A. "Doubling" of Local Photon Emissions from Two Simultaneous, Spatially Separated Chemiluminescent Reactions Share the Same Magnetic Field Configurations. J. Biophys. Chem. 2012, 3, 72-80.

37. Dotta, B.T.; Murugan, N.J.; Karbowski, L.M.; Persinger, M.A. Excessive Correlated Shifts in pH with Distal Solutions Sharing Phase-Uncoupled Angular Accelerating Magnetic Fields: Macro-Entanglement and Information Transfer. Int. J. Phys. Sci. 2013, 8, 1783-1787.

38. Karbowski, L.M.; Murugan, N.J.; Persinger, M.A. Experimentally-Induced Inhibition of Growth in Melanoma Cell Cultures Separated by $\sim 2$ Kilometers When Both Share Excess Correlation Magnetic Fields: Macroscopic Evidence of Free-Space Quantum Teleportation? J. Signal Inf. Process. 2015, 6, 39-48.

39. Lewis, W.C. A System of Physical Chemistry. Vol III. Quantum Theory; Longmans, Green and Company: Harlow, Essex, UK, 1914.

40. Persinger, M.A.; Koren, S.A. Dimensional Analyses of Geometric Products and the Boundary Conditions of the Universe: Implications for a Quantitative Value for the La-tency to Display Entanglement. Open Astron. J. 2013, 6, 10-13.

41. Wallace, L.; Bernath, P.; Livingtin, Q.; Hinkle, K.; Busler, J.; Guo, G.; Zhang, K. Water on the Sun. Science 1995, 268, 1155-1158.

42. Piccardi, G. The Chemical Bases of Medical Climatology; Charles C Thomas: Springfield, IL, USA, 1962.

43. Persinger, M.A.; Koren, S.A. Potential Role of the Entanglement Velocity of $10^{23} \mathrm{~m} \cdot \mathrm{s}^{-1}$ to Accommodate Recent Measurements of Large Scale Structures of the Universe. Int. Lett. Chem. Phys. Astron. 2015, 3, 106-112.

44. Pishchalnikov, R.; Pershin, S.M.; Bunkin, A.F. Quantum Differences in Ortho/para $\mathrm{H}_{2} \mathrm{O}$ Spin-Isomers as a Factor of the Femtosecond Charge Separation Kinetics Modulation in the Reaction Center of Purple Bacteria. Biophysics 2012, 57, 779-785.

(C) 2015 by the authors; licensee MDPI, Basel, Switzerland. This article is an open access article distributed under the terms and conditions of the Creative Commons Attribution license (http://creativecommons.org/licenses/by/4.0/). 